\title{
Komplexe Krankheiten brauchen mehr als nur Arzneien
}

\author{
Janssen-Cilag bietet seit Jahren mehr als nur innovative Arzneimittel. Mit \\ Delphi-Studien und Zukunftsarbeit versteht sich das Unternehmen als Motor \\ und Ideengeber für ein integriertes Gesundheitswesen. Geschäftsführer Hans \\ Wormann erklärt die Ziele der Unternehmenspolitik und aktuell anstehende \\ Projekte.
}

\begin{abstract}
MMW: Herr Wormann, mit dem Unternehmen Janssen-Cilag verbindet sich das Projekt „Zukunftsarbeit“ für die Gesundheitsversorgung. Mit welchen Ergebnissen? Wormann: Wir verstehen das seit 15 Jahren als Auftrag nach innen und nach außen. Für uns selbst heißt das: Bei der Entwicklung innovativer Medikamente wollen wir uns an der Gestaltung des gesamten Gesundheitssystems beteiligen. Dabei wollen wir nicht unter uns bleiben, sondern einen Dialog mit allen Beteiligten führen.
\end{abstract}

\section{MMW: Nimmt die Politik das auf?}

Wormann: Das wird sehr begrüßt, denn die Ergebnisse des Dialogs bündeln wir in unseren Delphi-Studien, von denen wir bereits fünf vorgelegt haben, jeweils zu aktuellen Fragen. Diese Ergebnisse stehen dann allen als Anregung zur Verfügung. So haben wir über Jahre zum Beispiel die Rolle und die Beteiligung des Patienten studiert und debattiert.

MMW: Was kommt in der Versorgung an? Wormann: Wir haben heute eine intensive Diskussion über das Management der Versorgungswirklichkeit. Früher hat man über Kostenmanagement gesprochen, heute über strukturelle Veränderungen, Kooperationen, neue Verantwortlichkeiten und Beteiligungen, mit dem Ziel besserer Behandlungsergebnisse.

MMW: Wie sehen die Pläne für Ihre Zukunftsarbeit in diesem Jahr aus?

Wormann: Nach Abschluss der Diskussion um das AMNOG wollen wir erneut Beteiligte des Gesundheitswesens einladen. Wir wollen dabei herausfinden: Wie muss in Deutschland ein ganzheitliches Versorgungs-Management für chronisch Kranke aussehen? Wie müssen die Prozesse der Versorgung gestaltet sein? Wer muss darin involviert werden?

MMW: Wie sehen Sie die Chance, mit Blick auf das geplante Versorgungsgesetz Vorschläge einzubringen?

Wormann: Wir werden einen runden Tisch ins Leben rufen. Da sitzen Ärzte, Apotheker, Wissenschaftler, Krankenkassen dabei; natürlich auch Patientenvertreter. Wir brauchen aber keinen, der uns die Welt schön redet. Wir brauchen auch Querdenker und Querdenken. Die gemeinsame Arbeit soll schon in diesem Jahr zu konkreten Ergebnissen führen.

MMW: Wie ist denn die Reaktion derer, die Sie zu diesem Diskussionsprozess einladen? Etwa bei Ärzten, von denen ein großer Teil Arzneimittelherstellern skeptisch gegenübersteht? Spüren Sie das?

Wormann: Durchaus. Beispielsweise in der Diskussion um die Frage, wer in neuen Systemen Steuerungsfunktion übernehmen soll. Unsere Antwort darauf ist eine stärkere Integration. Komplexe Krankheiten, die mit komplexen Medikamenten behandelt werden müssen, brauchen nicht nur ein wirksames Arzneimittel, sondern auch ein adäquates Patienten-Management, wie es das in klinischen Studien gibt. Wer dann dabei die Steuerung übernimmt, das kann man nicht pauschal beantworten. Janssen-Cilag ist aufgrund seiner Kompetenz bereit, gewisse Steuerungsfunktionen zu übernehmen und

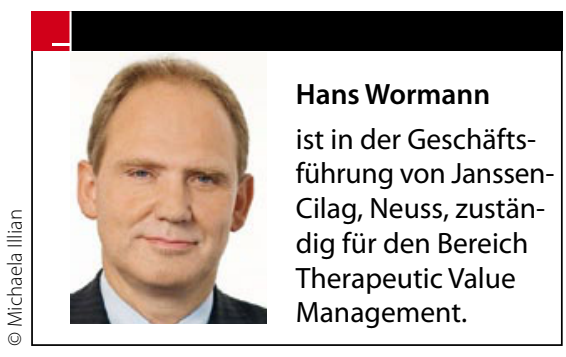

damit über die Rolle des reinen Arzneimittellieferanten herauszutreten.

MMW: Gibt es auch ein Problem mit der Fehlanwendung oder der nicht optimalen Anwendung von Arzneimitteln?

Wormann: Wir müssen noch mehr lernen, wie die Auswahl eines Arzneimittels für den spezifischen Patienten optimiert werden kann. Nicht jeder Patient spricht auf alle Behandlungen gleich an. Ein großes Ziel ist es, ein differenzierteres Bild zu bekommen, bis hin zur Entwicklung von Prädiktoren - ein wichtiger Schritt zur personalisierten Medizin.

MMW: Wie sehen aktuell Ihre Erfahrungen mit GBA und IQWiG aus?

Wormann: In der Vergangenheit haben wir festgestellt, dass - etwa bei Festbetragsverfahren - der Dialog sehr formell, sehr streng und eigentlich kein Dialog war. Dabei ist zu wenig differenziert worden, beispielsweise hinsichtlich der Effekte besonderer galenischer Formen. In klinischen Studien sind diese Vorteile, die aber im Alltag besonders relevant für Patienten sind, meist maskiert. Ich glaube, die Überzeugung wächst, dass wir uns auf einen lernenden Weg machen müssen. Diese Bereitschaft zum Dialog sehen wir auch beim GBA und beim IQWiG.

- Das Gespräch führten Wolfgang van den Bergh, Christoph Fuhr und Helmut Laschet. 